\title{
Consideraciones anestésicas en hipercapnia intraoperatoria, a propósito de un caso
}

\section{Case report: Anesthetic considerations in intraoperative hipercapnia}

Pedro Luis Quishpe Pila1, Andrea Carolina Pico Aguilar ${ }^{1}$

\begin{abstract}
Hypercapnia during the intraoperative period is one of the relevant conditions for the anesthesiologist, which can even condition the anesthetic technique, in case of an eventual complication. Where ventilatory monitoring and the interpretation of said disorder allows to diagnose, plan and treat the physiological consequences in the patient. We present the case of a 20 year old patient, scheduled for orthognathic surgery for diagnosis of mandibular body fracture, without added pathologies, no chest trauma, no ventilation disorders. It is presented with the objective of discussing the different considerations to be taken before the progressive establishment of hypercapnia, its causes, consequences and its management.
\end{abstract}

\section{RESUMEN}

La hipercapnia durante el intraoperatorio es una de las condiciones relevantes para el anestesiólogo, la cual puede incluso condicionar la técnica anestésica ante una eventual complicación. Donde la monitorización ventilatoria y la interpretación de dicho trastorno permite diagnosticar, planificar y tratar las consecuencias fisiológicas en el paciente. Presentamos el caso de una paciente de 20 años programada para cirugía ortognática por diagnóstico de fractura de cuerpo mandibular, sin patologías añadidas no traumas torácicos, no trastornos de ventilación. Se presenta con el objetivo de discutir las diferentes consideraciones a tomar ante la instauración progresiva de hipercapnia sus causas, consecuencias así como su manejo.

\section{Key words:}

Hypercapnia, exhaled $\mathrm{CO}_{2}$, orthognathic surgery, nasal intubation, anesthesia

\section{Palabras clave:}

Hipercapnia, $\mathrm{CO}_{2}$ exhalado, cirugía ortognática, intubación nasal, anestesia

1 Residente del Postgrado de Anestesiología de la Universidad San Francisco de Quito. Hospital Carlos Andrade Marín.

Fecha de recepción: 11 de diciembre de 2018

Fecha de aceptación: 24 de abril de 2019

\section{ORCID}

https://orcid.org/0000-0002-6406-6987

\section{Correspondencia:}

Pedro Luis Quishpe Pila

Email pedro_quishpe@yahoo.es/pedroq409@gmail.com 


\section{Introducción}

a hipercapnia se define generalmente como un nivel alto de dióxido de carbono $\left(\mathrm{CO}_{2}\right)$ mayor a $45 \mathrm{~mm}$ de $\mathrm{Hg}$, medida en sangre arterial[1]-[3], su monitoreo durante anestesia se puede realizar con la medición de capnometría o $\mathrm{CO}_{2}$ exhalado $\left(\mathrm{ETCO}_{2}\right)$, que puede diferir de la arterial en $\pm 5 \mathrm{~mm}$ de $\mathrm{Hg}[4],[5]$.

La incidencia o el desarrollo de esta alteración fueron variando según los años, sin embargo, se ha registrado un incremento aparente en la medida que se iban incorporando procedimientos más complejos, uso de $\mathrm{CO}_{2}$ externo y cada vez pacientes comprometidos en su estado físico[1],[6]-[9]. Se ha mantenido entre el $1 \%$ y el $3 \%$ de las complicaciones durante el transoperatorio, siendo las causas, la mayoría de veces predecibles y tratables, se considera como queja de diversas molestias en el postoperatorio inmediato en promedio 40 a un $60 \%$ de los pacientes presentan delirio, irritabilidad, náusea, vómito e incomodidad[10]-[13].

Durante el intraoperatorio la monitorización de $\mathrm{CO}_{2}$ exhalado $\left(\mathrm{ETCO}_{2}\right)>45 \mathrm{mmHg}$ se considera hipercarbia, la misma que conduce a cambios hemodinámicos como el aumento del gasto cardíaco, presión arterial media, liberación de catecolaminas, disminuyendo así la resistencia vascular sistémica por efecto directo del $\mathrm{CO}_{2}[1],[14]$, cuando no es contrarrestada existe una activación simpática con vasoconstricción, ocasionando trastornos en microdinamia que conduce a eventos deletéreos[15].

Los efectos fisiológicos de la hipercapnia se comprenden cada vez mejor, el conocimiento sobre los mecanismos de acción, sus causas y manejo, hacen más seguro un procedimiento quirúrgico, se asocian con la acidosis hipercápnica, que inhibe la ruta NF- $\mathrm{kB}$, explica varios efectos perjudiciales, incluida la demora de la cicatrización de las heridas y la disminución de la destrucción bacteriana[15]-[17].

\section{Caso clínico}

Paciente de 20 años, programada para cirugía ortognática, por fractura de cuerpo mandibular, sin alergias, sin historial de enfermedades pulmonares o cardiacas. Examen físico auscultación normal, retrognatia. Catalogada como riesgos quirúrgico moderado, equivalentes metabólicos (METS > 4), capacidad funcional (NYHA I), riesgo anestésico ASA I. Posibilidad de complicaciones pulmonares $0,1 \%$. Se obtiene consentimiento informado. La intervención quirúrgica se realiza con anestesia general e intubación nasal se colocó tubo preformado 6,0 con balón, por cerclaje mandibular, monitorización estándar de la Sociedad Americana de Anestesiología, hidratación con lactato ringer a $1 \mathrm{ml} / \mathrm{kg} / \mathrm{h}$.

En el intraoperatorio anestesia balanceada con remifentanilo $0,25 \mathrm{ug} / \mathrm{kg} / \mathrm{min}$ y sevofluorane CAM 0,5 , cambios en el monitoreo ventilatorio con elevación progresiva de $\mathrm{CO}_{2}$ exhalado hasta $55 \mathrm{~mm}$ de $\mathrm{Hg}$ (Figura 1), asociado, trastornos hemodinámicos con hipertensión, desaturación, hipoventilación, volumen tidal no alcanzado, toma de gasometría con $\mathrm{PaCO}_{2} 50$ $\mathrm{mmHg}$ más acidosis hipercápnica, continua con elevación de presión plateau hasta $38 \%$, por lo que se realiza un rastreo con fibroscopia encontrando tubo nasal doblado (Figura 2); se procede a cambio guiado por fibroscopia, colocándose tubo reforzado 6.0 con balón, procedimiento quirúrgico continúa sin mayor alteración. Se descarta posibilidad de hipertermia maligna.

Durante acto quirúrgico, canalización arterial y monitoreo invasivo, muestras arteriales tras cambio de tubo nasal y corrección de parámetros ventilatorios, demostró compensación ácido base $\mathrm{PaCO}_{2} 37$ $\mathrm{mmHg}$, sin mayores incidentes, se extuba, mantiene estabilidad.

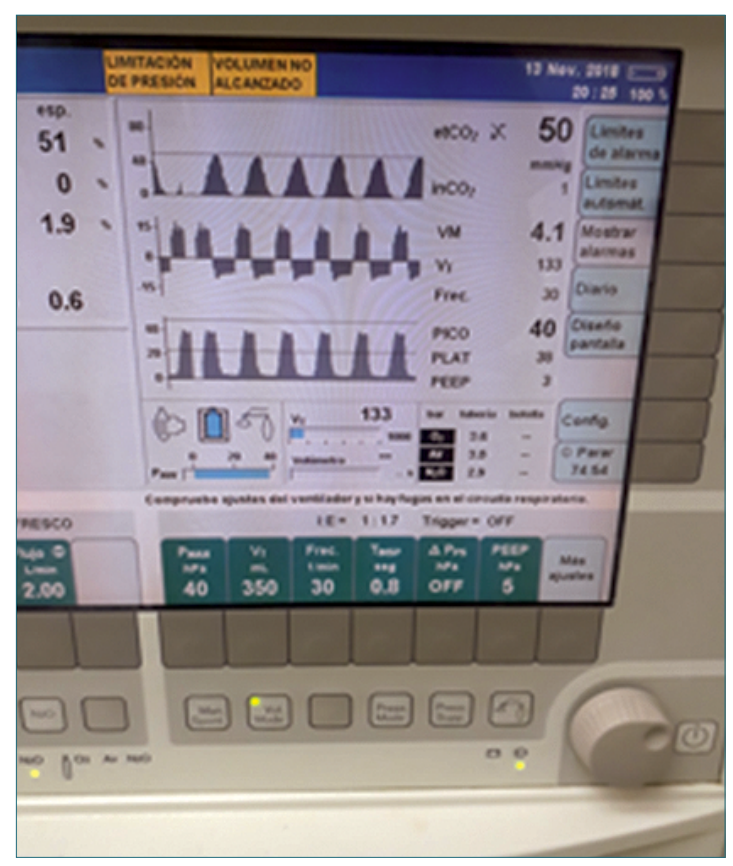

Figura 1. Máquina de anestesia apreciese $\mathrm{ETCO}_{2}$ hipercarbia y presión Plateu, volumen tidal insuficiente. Foto tomada por autor *HCAM

*HCAM. Hospital Carlos Andrade Marín. 


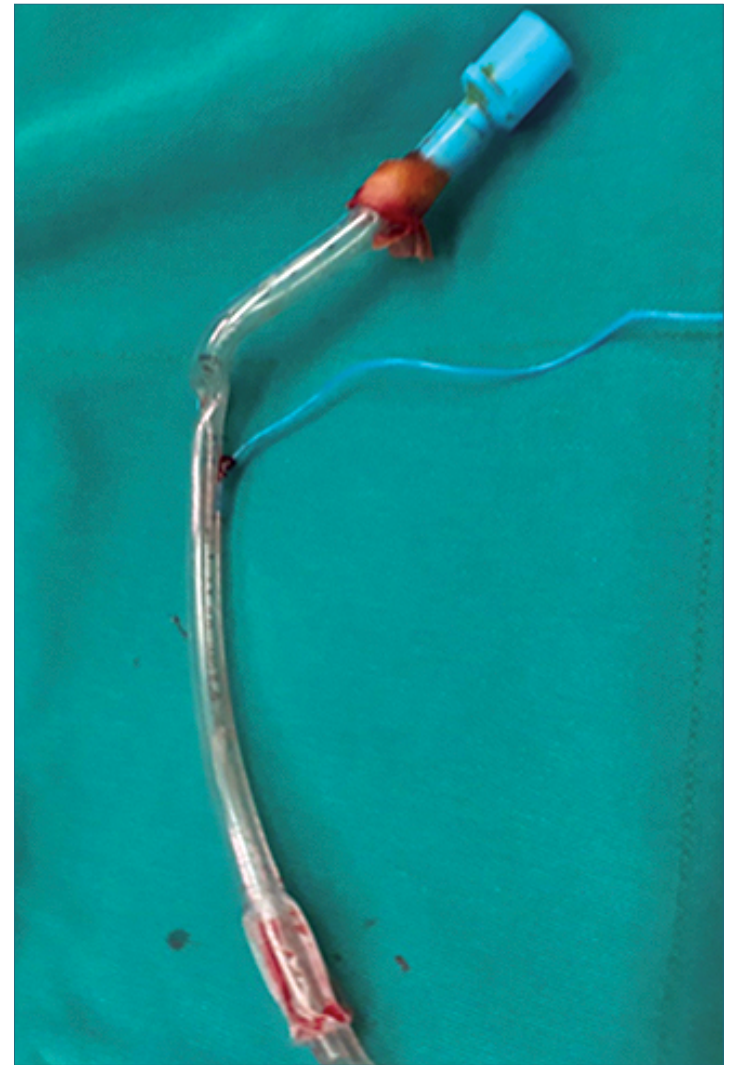

Figura 2. Tubo preformado nasal apréciese área doblada y rotado. Foto tomada por autor *HCAM.

*HCAM. Hospital Carlos Andrade Marín.

Postoperatorio en área monitorizada por 24 horas, controles de gasometría sin trastornos ácido base, neurológicamente sin alteraciones.

\section{Discusión}

La hipercapnia es el resultado del aumento de $\mathrm{CO}_{2}$ arterial que puede ser monitorizado durante el acto anestésico[1],[18],[19] con valores de $\mathrm{CO}_{2}$ exhalado $\left(\mathrm{ETCO}_{2}\right)$, hay que recordar valores normales de $\mathrm{CO}_{2}$ medidos en sangre arterial, hipocapnia $<35 \mathrm{mmHg}$, normocapnia 35-45 mmHg, hipercapnia $>45 \mathrm{mmHg}$, con el abordaje de ventilación mecánica se modifican los valores de $\mathrm{CO}_{2}$ exhalado $\pm 3-5 \mathrm{mmHg}$, su incremento muchas veces no es significativo en pacientes sanos, por períodos cortos[14],[15],[19].

La hipercapnia, es la consecuencia del descenso en la ventilación alveolar[15] produce trastornos hemodinámicos a nivel cardiovascular, cerebrovascular y pulmonar[14]. Su causa podría estar relacionada a fenómenos fisiopatológicos que desencadenan incremento de $\mathrm{CO}_{2}$ exhalado[6],[11],[17], debido:

1. Al incremento de espacio muerto: a) Disminución de la ventilación/perfusión (V/Q); b) Enfermedades pulmonares concomitantes; c) Alteraciones en la pared torácica[2].

2. Disminución en el volumen minuto: a) Obstrucciones; b) Malfuncionamiento de dispositivos; c) Procedimientos quirúrgicos[2].

3. Aumento en la producción de $\mathrm{CO}_{2}$ de forma continua: a) Sepsis; b) Convulsiones; c) Hipertermia maligna[2],[20].

El desarrollo de patologías que condicionan el incremento del espacio muerto debido a trastornos de ventilación y perfusión[4],[17],[21], se están volviendo cada vez más frecuentes, ya sea por obesidad, enfermos pulmonares crónicos o patologías que implican traumas torácicos. La fisiopatología se explica por el trastorno pulmonar acompañada de hipoventilación, ocasiona que disminuya la presión alveolar de oxígeno $\left(\mathrm{PAO}_{2}\right)$ y presión arterial de oxigeno $\left(\mathrm{PaO}_{2}\right)$ con retención de $\mathrm{CO}_{2}$ secundaria, este incremento de $\mathrm{CO}_{2}$ y trastornos $\mathrm{V} / \mathrm{Q}$, produce iones $\mathrm{H}$ que incrementa la $\mathrm{PaCO}_{2}$ volviendo los mecanismos compensatorios a la hipercapnia menos susceptible[9],[14],[18],[19]. En estos pacientes el abordaje de ventilación mecánica, para protección pulmonar, acepta un aumento de $\mathrm{CO}_{2}$ exhalado hasta $45 \mathrm{mmHg}$, el objetivo, minimizar la lesión pulmonar inducida por el ventilador, el mecanismo es útil en paciente enfermos crónicos, no está clara la asociación en trauma torácico, pero podría beneficiarse, siempre con un monitoreo de microdinamia, control de gases arteriales y prevención del desarrollo de acidosis hipercápnica[9],[11],[12],[19].

Aquellos trastornos asociados a disminución de volumen minuto, el monitoreo y el conocimiento de las ondas de capnografia es importante[15],[19]. Datos como una curva de capnografía característica, junto a un volumen minuto bajo y presiones altas de la vía aérea, suponen obstrucción. La fisiopatología se explica por la obstrucción del tubo traqueal o complementos, esto desencadena dismución del compliance pulmonar y trastornos en ventilación/perfusión, incrementando $\mathrm{CO}_{2}$ exhalado con advenimiento de hipercapnia, con este patrón indicado es necesaria una confirmación y funcionalidad de instrumentos, descartar las posible alteraciones previene eventos adversos[10],[15],[22].

El incremento súbito de $\mathrm{CO}_{2}$ puede deberse en gran parte a un estado hiperdinámico, catabólico que producen cargas altas de $\mathrm{CO}_{2}$, ya sea por alteraciones 
tales como sepsis o convulsiones, genéticas como el desarrollo de hipertermia maligna, la fisiopatología en estados hiperdinámicos, incrementa los requerimientos de oxígeno, produce hiperventilación, con incremento de la $\mathrm{PaO}_{2}$, desproporción V/Q y cargas altas de $\mathrm{CO}_{2}$ que a vez, conducen a hipoxemia, hipercapnia y colapso cardiovascular[7],[15],[19],[20]. El manejo en estos pacientes tiene que ser exhaustivo, control seriado de gases arteriales, ventilación mecánica protectiva y limitar los causantes del desarrollo de tales patologías[7].

La hipercapnia, a nivel pulmonar, modula la resistencia de las vías respiratorias, a nivel cardiovascular, incrementa la liberación de catecolaminas con impacto sobre la función cardiaca modificando el flujo arterial y venoso, a nivel cerebrovascular modifica el flujo cerebral. Todos estos efectos tienen impacto sobre el estado hemodinámico del paciente[3],[7], produciendo, entre otros efectos, superficialización del plano anestésico, despertar intraoperatorio, dificultades en la extubación[5]. Un punto necesario a recordar es la asociación de hipercapnia, con el desarrollo concomitante de acidosis hipercápnica, $\left(\mathrm{pCO}_{2}>50 \mathrm{con} \mathrm{pH}<\right.$ $7,26)$ cuyos efectos tienen repercusión sobre la microdinamia, se lo ha asociado con inhibición de la ruta
NF- $\kappa B$ del estado inmunitario, por lo que presentan alteraciones en la cicatrización y destrucción bacteria$\mathrm{na}[7],[10],[20]$.

\section{Conclusiones}

La hipercapnia intraoperatoria puede influir significativamente sobre el estado hemodinámico del paciente con aumento del gasto cardiaco o alteraciones descritas, si bien tiene factores a favor y en contra, por la concomitancia de la acidosis hipercápnica que produce efectos nocivos, el estricto monitoreo y la compensación siempre tiene que ser valorada de acuerdo a las condiciones del paciente.

Todo incremento de $\mathrm{CO}_{2}$ exhalado $\left(\mathrm{ETCO}_{2}\right)$ debe generar cuestionamientos respecto a su causa, hay que considerar problemas ventilatorios mecánicos (equipo y dispositivos), descartarse problemas del paciente (enfermedad pulmonar o cardiaca), considerar problemas quirúrgicos, incluidos trastornos genéticos. El conocimiento de la fisiopatología de estos trastornos que producen hipercarbia es esencial para comprender mejor su causa, su repercusión clínica y las posibles opciones de compensación

\section{Referencias}

1. Obuchi T, Shimamura $\mathrm{S}, \mathrm{Mi}-$ yahara N, Fujimura N, Iwasaki A. CO2 retention: the key to stopping hiccups. Clin Respir J. 2018 Aug;12(8):2340-5. https:// doi.org/10.1111/crj.12910 PMID:29729130

2. Farina S, Bruno N, Agalbato $C$, Contini M, Cassandro R, Elia D, et al. Physiological insights of exercise hyperventilation in arterial and chronic thromboembolic pulmonary hypertension. Int J Cardiol. 2018 May;259(259):178-82. https://doi.org/10.1016/j. ijcard.2017.11.023 PMID:29579597

3. Kanaya A, Kuratani N, Kaiho Y, Yamauchi M. Intraoperative hypercapnia and anesthesia emergence after pediatric ambu- latory surgery. Paediatr Anaesth. 2016 Nov;26(11):1116-7. https:// doi.org/10.1111/pan.12984 PMID:27747982

4. Lam T, Nagappa M, Wong J, Singh $\mathrm{M}$, Wong $\mathrm{D}$, Chung $\mathrm{F}$. Continuous Pulse Oximetry and Capnography Monitoring for Postoperative Respiratory Depression and Adverse Events: A Systematic Review and Meta-analysis. Anesth Analg. 2017 Dec;125(6):201929. https://doi.org/10.1213/ ANE.0000000000002557 PMID:29064874

5. Mao QX, Guo W, Huang BQ, Yan $\mathrm{H}$. Impact of artificial capnothorax on coagulation in patients during video-assisted thoracoscopic esophagectomy for squamous cell carcinoma. Surg Endosc. 2016 Jul;30(7):2766-72. https://doi.org/10.1007/s00464-
015-4549-3 PMID:26563508

6. Harbut P, Gozdzik W, Stjernfält E, Marsk R, Hesselvik JF. Continuous positive airway pressure/ pressure support pre-oxygenation of morbidly obese patients. Acta Anaesthesiol Scand. 2014 Jul;58(6):675-80. https:// doi.org/10.1111/aas. 12317 PMID:24738713

7. Contreras M, Masterson C, Laffey JG. Permissive hypercapnia: what to remember. Curr Opin Anaesthesiol. 2015 Feb;28(1):2637. https://doi.org/10.1097/ ACO.0000000000000151 PMID:25500498

8. Longo MA, Cavalheiro BT, de Oliveira Filho GR. Laparoscopic cholecystectomy under neuraxial anesthesia compared with general anesthesia: systematic review and meta-analyses. J Clin Anesth. 2017 Sep;41:48-54. 
https://doi.org/10.1016/j.jclinane.2017.06.005 PMID:28802605

9. Fritsch S, Bickenbach J. [Respiratory Insufficiency: State of the Art - Diagnosis and Therapy]. Anasthesiol Intensivmed Notfallmed Schmerzther. 2018 Feb;53(2):90-101. https://doi. org/10.1055/s-0043-107167 PMID:29426048

10. Dunworth SA, Natoli MJ, Cooter $M$, Cherry AD, Peacher DF, Potter JF, et al. Hypercapnia in diving: a review of $\mathrm{CO}_{2}$ retention in submersed exercise at depth. Undersea Hyperb Med. 2017 May-Jun;44(3):191-209. https:// doi.org/10.22462/5.6.2017.1 PMID:28779577

11. Meleiro H, Correia I, Charco Mora P. New evidence in one-lung ventilation. Rev Esp Anestesiol Reanim. 2018 Mar;65(3):149-53. https://doi. org/10.1016/j.redar.2017.06.007 PMID:28967439

12. Quintero MC, Putnam RW, Cordovez JM. Theoretical perspectives on central chemosensitivity: $\mathrm{CO} 2 / \mathrm{H}+$-sensitive neurons in the locus coeruleus. PLOS Comput Biol. 2017 Dec;13(12):e1005853. https://doi.org/10.1371/journal. pcbi. 1005853 PMID:29267284

13. Geurts LJ, Bhogal AA, Siero JC, Luijten PR, Biessels GJ, Zwanenburg JJ. Vascular reactivity in small cerebral perforating arteries with $7 \mathrm{~T}$ phase contrast MRI - A proof of concept study. Neuroimage. 2018 May;172(172):470-7. https:// doi.org/10.1016/j.neuroimage.2018.01.055 PMID:29408324
14. Lee JE, Kim JH, Kim SO. Misinterpretation of carbon dioxide monitoring because of deadspace of heat and moisture exchanger with a filter in pediatric anesthesia: A case report. Medicine (Baltimore). 2018 Aug;97(35):e12158. https://doi.org/10.1097/ MD.0000000000012158 PMID:30170460

15. Hodgson C, Cooper DJ, Arabi $Y$, Bennett V, Bersten A, Brickell K, et al.; PHARLAP Study Investigators and the Australian and New Zealand Intensive Care Society Clinical Trials Group. Permissive Hypercapnia, Alveolar Recruitment and Low Airway Pressure (PHARLAP): a protocol for a phase 2 trial in patients with acute respiratory distress syndrome. Crit Care Resusc. 2018 Jun;20(2):139-49. PMID:29852853

16. Wang J, Li Z, Yu Y, Li B, Shao G, Wang Q. Risk factors contributing to postoperative delirium in geriatric patients postorthopedic surgery. Asia-Pac Psychiatry. 2015 Dec;7(4):375-82. https:// doi.org/10.1111/appy.12193 PMID:26097170

17. Raveendran R, Wong J, Singh M, Wong DT, Chung F. Obesity hypoventilation syndrome, sleep apnea, overlap syndrome: perioperative management to prevent complications. Curr Opin Anaesthesiol. 2017 Feb;30(1):146-55. PMID:27792079

18. Kitajima $T$, Marumo S, Shima H, Shirata M, Kawashima S, Inoue
D, et al. Clinical impact of episodic nocturnal hypercapnia and its treatment with noninvasive positive pressure ventilation in patients with stable advanced COPD. Int J Chron Obstruct Pulmon Dis. 2018 Mar;13:843-53. https://doi.org/10.2147/COPD. S153200 PMID:29563784

19. Brat K, Plutinsky M, Hejduk K, Svoboda M, Popelkova P, Zatloukal J, et al. Respiratory parameters predict poor outcome in COPD patients, category GOLD

2017 B. Int J Chron Obstruct Pulmon Dis. 2018 Mar;13:1037-52. https://doi.org/10.2147/COPD. S147262 PMID:29628761

20. Schneiderbanger $D$, Johannsen S, Roewer N, Schuster F. Management of malignant hyperthermia: diagnosis and treatment. Ther Clin Risk Manag. 2014 May;10:355-62. https:// doi.org/10.2147/TCRM.S47632 PMID:24868161

21. El Jaouhari SD, Mamane Nassirou O, Meziane M, Bensghir M, Haimeur C. [Gas tamponade following intraoperative pneumothorax on a single lung: A case study]. Rev Pneumol Clin. 2017 Apr;73(2):90-5. https://doi.org/10.1016/j. pneumo.2017.01.004 PMID:28259372

22. Choi YH, Lee DH. A Rare Airway Obstruction Caused by Dissection of a Reinforced Endotracheal Tube. J Emerg Med. 2018 Apr;54(4):e73-5. https://doi.org/10.1016/j. jemermed.2017.12.043 PMID:29395694 